\title{
Regiones administrativas y de planificación: análisis comparativo de las primacías urbanas en la RAPE Región Central y RAP Pacífico
}

\author{
Regiões administrativas e de planejamento: análise comparada das primazias \\ urbanas na RAPE Região Central e RAP Pacífico
}

Administrative and planning regions: comparative analysis of urban primacies in the Central Region - RAPE and Pacifico - RAP

Melba Rubiano-Bríñez[a] (1), César Eligio-Triana[a] (D)

[a] Universidad Piloto de Colombia, Bogotá, Colombia

Cómo citar: Rubiano-Bríñez, M., \& Eligio-Triana, C. (2019). Regiones administrativas y de planificación: análisis comparativo de las primacías urbanas en la RAPE Región Central y RAP Pacífico. urbe. Revista Brasileira de Gestão Urbana, 11, e20180107. https://doi.org/10.1590/2175-3369.011.e20180107

\section{Resumen}

El estudio del ordenamiento territorial analiza variables y escalas en las cuales se observa el uso y ocupación del territorio, y cómo las decisiones adoptadas inciden sobre la población y las condiciones de vida en relación a otras áreas del país. Dado el carácter heterogéneo del territorio colombiano, y a pesar de la existencia de directrices y de una Ley Orgánica de Ordenamiento Territorial, resulta una labor compleja establecer un modelo a aplicar en todo el país, lo que denota la existencia de primacías urbanas. Metodológicamente se abordan conceptos relacionados con las primacías urbana, focalizándose en la Región Administrativa y de Planificación Especial (RAPE Región Central) y la Región Administrativa y de Planificación (RAP Pacífico), a partir de la información disponible en el Departamento Administrativo Nacional de Estadística (DANE), se propone la cuantificación a través de indicadores de primacías (variables poblacionales, económicas y estatales). Este análisis permitió identificar desequilibrios y establecer comparaciones entre la RAPE-RC y la RAP-P, identificando la presencia de macrocefalias urbanas generadas en Bogotá y Cali, tendencias constantes en la ocupación del territorio y algunas variaciones en las ciudades de las posiciones inferiores de los índices primaciales. También se identificó la diversidad en la configuración de las RAP, lo cual acentúa los desequilibrios, y permitió observar oportunidades en la construcción de instrumentos de ordenamiento del territorio e incentivos que permitan cerrar las diferencias existentes.

Palabras clave: Contraurbanización. Desequilibrio regional. Indicadores urbanos. Macrocefalia urbana. Ordenamiento territorial.

\section{Resumo}

O estudo do ordenamento territorial analisa variáveis e escalas nas quais observa-se o uso e ocupação do território e como as decisões afetam a população e as condições de vida em relação às outras áreas do país. Dado a heterogeneidade do território colombiano, e apesar da existência de diretrizes e de uma Lei Orgânica sobre Ordenamento Territorial, é uma tarefa complexa estabelecer um modelo a implementar

MRB es economista, magíster en Planeamiento Urbano y Regional, candidata a doctora en Geografía, Investigadora junior Colciencias, email: melba-rubiano@unipiloto.edu.co

CET es arquitecto, magíster en Arquitectura, investigador junior Colciencias, representante Andes Norte Asociación de Revistas Latinoamericanas de Arquitectura (ARLA), e-mail: cesar-eligio@upc.edu.co; cesareligioarq@gmail.com 
que denote em todo o país a existência de primazias urbanas. Metodologicamente, abordam-se conceitos relacionados com as primazias urbanas, focalizado na Região Administrativa e de Planejamento Especial (RAPE Região Central) e a Região Administrativa e de Planejamento (RAP Pacífico), a partir da informação disponível no Departamento Administrativo Nacional de Estatística (DANE), onde se propõe a quantificação através de indicadores de primazias (variáveis populacionais, econômicas e estatais). Esta análise mostrou desequilíbrios, estabeleceu comparações entre a RAPE-RC e a RAP-P, identificou a presença de macrocefalias urbanas geradas em Bogotá e Cali, tendências constantes na ocupação do território e algumas variações nas cidades das posições mais baixas dos índices. Identificou-se a diversidade na configuração das RAP que acentua os desequilíbrios e permitiu observar uma potencialidade para construir instrumentos de ordenamento territorial e incentivos para fechar as diferenças existentes.

Palavras-chave: Contra urbanização. Desequilíbrio regional. Indicadores urbanos. Macrocefalia urbana. Ordenamento territorial.

\section{Abstract}

Territorial planning implies the examination of variables such as land use, land occupation patterns and the impacts of institutional decision making on the population's living conditions. Due to Colombia's territorial heterogeneity, it is rather difficult to implement territorial "blueprint" models throughout the country in which there is a clear primacy of urban centers. This paper draws on urban primacy indicators, particularly focused on two Administrative Planning Region cases: "Central Region - RAPE (CR-RAPE)" and "Pacific Region - RAP (PR-RAP)". Additionally, based on official information provided by the National Administrative Department of Statistics (DANE), the paper analyzes ways for RAP's framework implementation and impact evaluation by assessing population, economics and governmental variables. By comparing CR-RAPE and PR-RAP, the analysis allowed to identify territorial unbalances particularly triggered by urban macrocephaly phenomenon in Bogotá and Cali, constant trends in land occupation and non-significant variations in primacy indicators in the rest of the cities encompassing each analyzed RAP. Finally, the study allowed to recognize the territorial diversity of each RAP, which in one hand exacerbates the aforementioned unbalances, but on the other hand, raise awareness about the opportunity for designing territorial planning tools and incentives aiming at bridging gaps among the territories.

Keywords: Counter urbanization. Regional imbalance. Urban indicators. Urban macrocephaly. Territory management.

\section{Introducción}

El proceso de urbanización de la ciudad latinoamericana está caracterizado por altos grados de concentración urbana (demográfica y económica) que las ciudades capitales tienen respecto a su propia red. Este fenómeno urbano generó que los estudios de las ciudades, tengan muchas interpretaciones que cuestionan la manera cómo se afrontan y construyen los lineamientos del ordenamiento territorial. Diversos autores han introducido conceptos emergentes con el fin de orientar la compresión de las nuevas realidades urbanas y los instrumentos de gestión urbana en marcos interdisciplinares (Llano, 2018).

Los "desarrollos geográficos desiguales" sirven de fundamento teórico para analizar las formas de las aglomeraciones y la conformación de regiones, que siguen favoreciendo a unas en detrimento de otras y como destaca Harvey (2014, p. 162) dejan en evidencia los "[...] desplazamientos de los fallos sistémicos del capital de un lugar a otro".

En este sentido, se retoman aportes desde la geografía para comprensión de conceptos como: espacio geográfico, territorio y región. Lobato Correa (1998) señala que el espacio geográfico es multidimensional, Haesbaert (2014), considera que ese espacio geográfico es la dimensión espacial de la sociedad. En relación al 
territorio, Buzai (2014), destaca la importancia de la geografía como ciencia de la organización del territorio. Coraggio (1987), plantea la región como una porción del territorio donde tienen lugar procesos y relaciones sociales, donde existen elementos y procesos naturales que están articulados y conforman el complejo social natural, el tamaño, forma y localización de la región responderá a la lógica de los procesos sociales, y esta determinará el tipo de organización espacial, así como las formas de apropiación del territorio. En términos de lo antes señalado, George (1967, p. 326-327) define:

Una región constituye sobre la tierra un espacio preciso, pero no inmutable, inscrito en un marco natural dado, y que responde a tres características esenciales: los vínculos existentes entre sus habitantes, su organización en torno a un centro dotado de una cierta autonomía, y su integración funcional en una economía global. Es el resultado de una asociación de factores activos y pasivos de intensidades variables, cuya dinámica propia se encuentra en el origen de los equilibrios internos y de la proyección espacial.

En esta definición de George se percibe la dinámica de la región y el rol de la población. Así mismo, considera que para llevar a cabo un estudio regional se deben tener en cuenta factores (variables): demográficos, naturales, productivos y de infraestructura.

Otras teorías y conceptos como Metápolis (Ascher, 1995, 2010), Postmetropolis ${ }^{1}$ (Soja, 2008), ponen de manifiesto la crisis y las posibles consecuencias de continuar gestionando las ciudades como tradicionalmente se hace, con esto se busca generar alternativas de compresión y solución, para orientar el entendimiento de la naturaleza inherentemente regional de la urbanización y de la acción social que en ella se desarrolla.

Adicionalmente, se puede afirmar que, el territorio colombiano se reconoce a partir de regiones, Pacífico, Caribe, Andina, Orinoquia y Amazonia (Figura 1a), estas describen con mayor exactitud la realidad del territorio y están en armonía con el espacio geográfico. En este marco, la primacía y el desequilibrio son hechos constantes que debe atender el proceso de planificación urbana. Los estudios institucionales sobre ordenamiento territorial, dan cuenta de la existencia de diferentes escalas en dicha forma de organización jurisdiccional y, por ende, implican que la comprensión del territorio debe abordarse desde diferentes escalas y jerarquías dada la heterogeneidad del país. Si las condiciones de las regiones fuesen homogéneas no existirían desequilibrios y la construcción del modelo de ordenamiento territorial no sería conflictivo ni complejo.

En este sentido, la investigación sobre el análisis de las primacías parte del reconocimiento de estas diferencias, con el fin de entender las implicaciones de los desequilibrios sobre la búsqueda del poder sobre el territorio. A partir de la creación de Regiones Administrativas y de Planificación (RAP), es el propósito de este artículo, presentar un análisis comparado entre la Región Administrativa y de Planificación Especial RAPE Región Central y la Región Administrativa y de Planificación RAP Pacífico, a partir del cálculo de los índices primaciales en términos poblacionales, económicos y de estatalidad.

Así, bajo la idea que el Ordenamiento Territorial es eminentemente político y siguiendo el modelo de El Estado estratega para el ordenamiento territorial de Revéiz (2013), la utilidad de este tipo de investigaciones se inscribe en la búsqueda de contenidos y estrategias de una Política Nacional de Ordenamiento Territorial.

Por otra parte el Gobierno Nacional en el Plan Nacional de Desarrollo (2010-2014) "Prosperidad para Todos" (DNP, 2010) ${ }^{2}$ dio prioridad al desarrollo de las ciudades y regiones para lograr el cumplimiento de las políticas sociales y de productividad buscando superar el relativo distanciamiento del nivel nacional con los temas específicos de las ciudades desde el proceso de descentralización (DNP, 2014a, p. 8). En este contexto es importante destacar que el interés que se genera por

\footnotetext{
${ }^{1}$ El concepto de sinecismo tiene un alcance implícitamente regional. Concierne no sólo a un centro urbano singular y densamente poblado sino, más categóricamente, a un sistema regional policéntrico más grande de asentamientos nodales interactivos, una ciudad región (Soja, 2008, p. 46)

${ }^{2}$ Capítulo III-Crecimiento sostenible y competitividad.
} 
categorías como distancia y densidad (aglomeración) de las actividades económicas y barreras que impiden su desarrollo (fronteras, conectividad, etc.) son los nuevos elementos que deben considerarse en los programas y políticas de desarrollo (DNP, 2014a, p. 8).

estos aspectos evidencian la presencia de primacías urbanas y a su vez como el ordenamiento territorial prima y privilegia las grandes aglomeraciones urbanas.

La anterior reflexión suscita los siguientes interrogantes ¿Cómo el ordenamiento territorial puede contribuir a atenuar los desequilibrios territoriales? ¿Cuáles son las relaciones primaciales entre las ciudades de una red urbana? ¿Qué factores tienen mayor incidencia en conformación de las primacías urbanas? ¿Cómo las primacías aportan en los desequilibrios territoriales?

Con el propósito de responder a estos cuestionamientos se plantea como hipótesis que, la fragilidad de lineamientos en el ordenamiento territorial, evidencian un polimetropolitanismo o una macrocefalia urbana que repercute en desequilibrios territoriales y estos inciden sobre la forma de ocupación del territorio.

El libro Misión sistema de ciudades. Una política nacional para el sistema de ciudades colombiano con visión a largo plazo, (DNP, 2014a) plantea una visión del territorio con miras a generar una mejor integración basada en 6 aspectos básicos

1. Visión Sostenible: reconocer y planear en función del Ordenamiento del Territorio;

2. Coordinación: Facilitar el trabajo conjunto entre las entidades territoriales;

3. Financiación eficiente: Identificar instrumentos para financiar actuaciones urbanas;

4. Productividad: Fortalecer los factores locales que promueven la productividad del Sistema de Ciudades;

5. Conectividad: Mejorar la conectividad en el Sistema de Ciudades;

6. Calidad de Vida: Disminuir las brechas sociales entre aglomeraciones urbanas.

Valorar estos 6 componentes también puede ser la base para la revisión de las incidencias del ordenamiento territorial en las primacías urbanas existentes y cómo superar las barreras físicas y administrativas.

Un antecedente de la evaluación de los desequilibrios territoriales presentes en la Región Administrativa y de Planeación Especial, Región Central (RAPE-RC), afirma que:

Para que este instrumento de gestión y planificación alcance los objetivos de integración regional y de competitividad, debe basarse en el conocimiento del contexto y las dinámicas de los territorios que lo componen [...] A partir de la información de densidad poblacional, los índices de rango-tamaño, de primacía y Clark-Evans, y los porcentajes de ocupación por sectores productivos y especialización de las capitales departamentales de la RAPE-RC, se realizó la descripción de los componentes de la estructura y la función del sistema de ciudades, la cual presenta una distribución vertical desequilibrada, con un sistema macrocéfalo concentrado en el Distrito Capital y una diversificación en la especialización de las actividades de las principales ciudades (González Pinto \& Solorza Bejarano, 2017, p. 23).

Los resultados de este estudio aportan aspectos metodológicos y reafirman la idea de un desequilibrio latente.

\section{Ordenamiento territorial a partir de la conformación de RAP (Región Administrativa y de Planeación)}

Las recientes orientaciones sobre el ordenamiento territorial apuntan a la consolidación de regiones como alternativa a la centralización. Acorde con esto, el artículo 30 de la Ley 1454 de 2011 establece que

[...] las Regiones Administrativas y de Planificación (RAP) son entidades conformadas por dos o más departamentos, las cuales cuentan con personería jurídica, autonomía financiera y patrimonio propio, cuya finalidad está orientada al desarrollo regional, la inversión y la competitividad, en los 
términos previstos en el artículo $306^{3}$ de la Constitución Política de Colombia y en el marco de los principios consagrados en la misma ley (Colombia, 2011, Art. 30).

En este sentido, las RAP se entienden como un instrumento de planeación a largo plazo que permite gerenciar y gestionar proyectos pertinentes para la región.

Actualmente en Colombia se encuentran conformadas tres RAP, la Región Administrativa y de Planeación Especial (RAPE Región Central); la Región Administrativa y de Planificación - RAP Pacífico y la RAP Caribe 4 . Además, se anunció la creación de la RAP Eje Cafetero (Caldas, Risaralda y Quindío). Para este estudio, se tomarán la Región Central y la Región Pacífica (Figura 1b), debido a que la primera concentra la mayor cantidad de población e incluye la capital del país y la segunda porque abarca una diversidad de departamentos con características muy heterogéneas entre sí, que permiten identificar las diferentes ocupaciones del territorio colombiano.

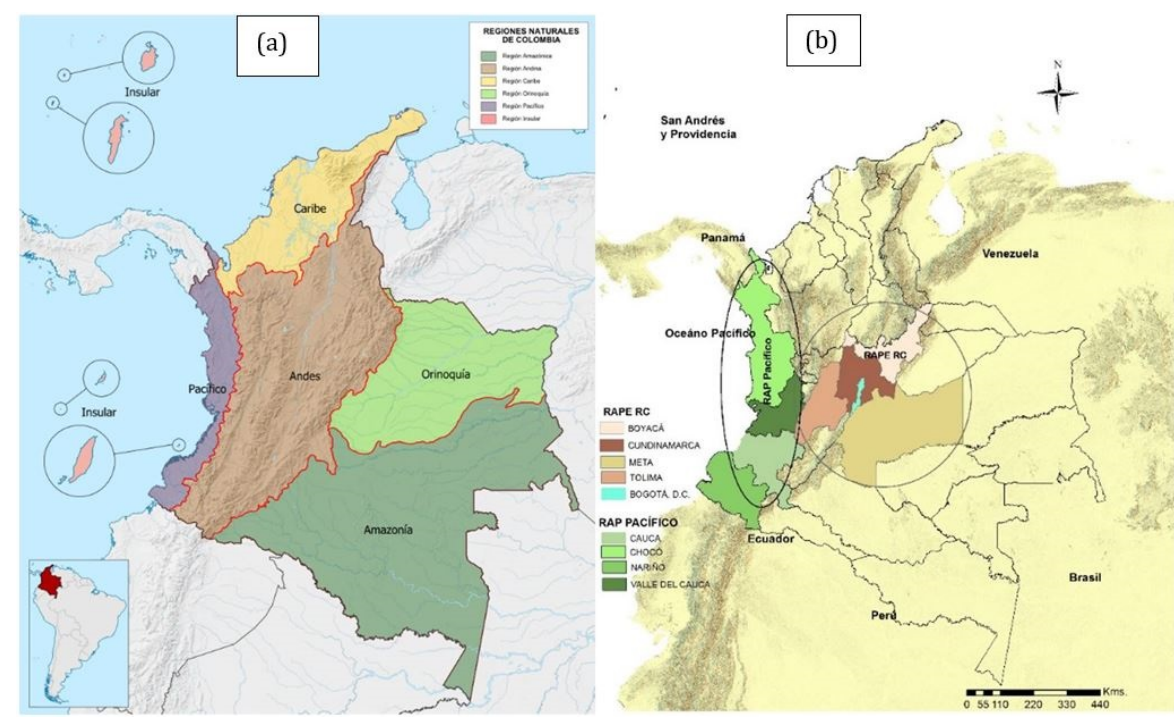

Figura 1 - Localización geográfica en el territorio colombiano de las regiones naturales (a) y de la RAPE Región Central y RAP Pacífico (b). Fuente: (a) Shadowxfox (2014); (b) Elaboración propia (2018).

Los atributos de cada RAP se sintetizan en la (Tabla 1), en ella se ponen de manifiesto la importancia que estas tienen en la conformación del territorio colombiano al generar el 55\% del PIB, contener el 46\% de la población y ocupar el $51 \%$ del territorio nacional.

Tabla 1 - Conformación de las RAP Central y Pacifico

\begin{tabular}{ccc}
\hline & RAPE-Región Central & RAP-Pacifico $^{5}$ \\
\hline Documentos de creación & Acuerdo Regional No 001 & Ordenanza No. 418 \\
Fecha de conformación & Convenio No. 1676 de 2014 & Julio 13 de 2016 \\
Territorio & 30 de septiembre de 2014 & $22 \%$ \\
Población & $29 \%$ & $17 \%-8.151 .955$ habitantes \\
Departamentos & $29 \%-13.870 .477$ habitantes \\
Capitales & 4 - Cundinamarca, Boyacá, Meta y Tolima & $4-$ Valle, Nariño, Cauca y Chocó \\
Municipios & Bogotá, Tunja, Villavicencio, lbagué & Cali, Pasto, Popayán Quibdó \\
PIB & $30 \%-316$ & $16 \%-178$ \\
& $40 \%$ & $15 \%$ \\
\hline
\end{tabular}

Fuente: Elaboración propia (2017) a partir de información disponible sobre las RAP. Región Central (2004) y RAP-Pacifico (2018).

\footnotetext{
${ }^{3}$ Dos o más departamentos podrán constituirse en regiones administrativas y de planificación, con personería jurídica, autonomía y patrimonio propio. Su objeto principal será el desarrollo económico y social del respectivo territorio (Colombia, 1991, art. 306).

${ }^{4}$ Acuerdo de integración se firmó el 19 de octubre de 2017.

${ }^{5}$ Junto con la Región Caribe, acusan el mayor Índice de Necesidades Básicas Insatisfechas (NBI) del país.
} 


\section{Metodología}

Se plantea una investigación en diferentes niveles, el primero, de carácter descriptivo y comparativo en cual se tomaron datos del Departamento Administrativo Nacional de Estadística (DANE), los cuales fueron observados en una perspectiva histórica con el fin de identificar estacionalidades, rupturas y cambios. El segundo, se abordó desde lo explicativo y correlacional, para identificar la asociación entre los desequilibrios territoriales y el ordenamiento territorial.

El tema de estudio tiene un enfoque cuantitativo y los datos obtenidos se interpretaron mediante un análisis estadístico. La investigación se llevó a cabo mediante la recopilación y análisis de datos asociados a variables de factores poblacionales, económicos y estatales.

\section{Indicadores de primacía}

La idea de productividad y la relación con la de costos de la vida (DNP, 2014a, p. 13) genera un primer modelo para valorar la concentración urbana en términos de equilibrio interno, ya que redundan en un mayor estado de bienestar y eficiencia económica.

El texto Urbanization and Spatial Structure Evolution of Urban System in China (Zhuoyong, 2008) y las aproximaciones de Cuervo González (2004), plantean una aproximación a la manera de medir este fenómeno urbano a partir de los indicadores de primacía. Zhuoyong expone el cálculo de $\mathrm{S}_{2} \mathrm{~S}_{4}$ y $\mathrm{S}_{11}$ (Tabla 2):

Table 8 depicts each province's urban primacy index (S2), four city index (S4) and eleven city index (S11) which were often employed in the study of city-size distribution. They can be written as:

$$
\begin{aligned}
& \mathrm{S} 2=\mathrm{P} 1 / \mathrm{P} 2 \\
& \mathrm{~S} 4=\mathrm{P} 1 /(\mathrm{P} 2+\mathrm{P} 3+\mathrm{P} 4) \\
& \mathrm{S} 11=2 \mathrm{P} 1 /(\mathrm{P} 2+\mathrm{P} 3+\ldots \ldots+\mathrm{P} 11) .
\end{aligned}
$$

\begin{tabular}{|c|c|c|c|c|c|c|c|}
\hline Provincia & $S_{2}$ & $S_{4}$ & $S_{11}$ & Provincia & $S_{2}$ & $\mathrm{~S}_{4}$ & $S_{11}$ \\
\hline $\begin{array}{c}\text { Beijing \& Tianjin \& } \\
\text { Hebei }\end{array}$ & 1,79 & 1,10 & 1,41 & Hunan & 2,55 & 0,87 & 0,72 \\
\hline Hebei & 1,38 & 0,57 & 0,57 & Guangdong & 1,23 & 0,63 & 0,80 \\
\hline Shanxi & 2,43 & 1,30 & 1,36 & Guangxi & 1,39 & 0,62 & 0,67 \\
\hline Mongolia Interior & 1,42 & 0,75 & 0,79 & Hainan & 2,40 & 1,19 & - \\
\hline Liaoning & 1,55 & 0,79 & 0,86 & $\begin{array}{c}\text { Chongqing \& } \\
\text { Sichuan }\end{array}$ & 1,49 & 1,10 & 1,35 \\
\hline Jilin & 1,97 & 1,19 & 1,25 & Sichuan & 5,70 & 2,02 & 1,57 \\
\hline Heilongjiang & 2,63 & 1,09 & 0,99 & Chongqing & 19,38 & 7,30 & - \\
\hline $\begin{array}{c}\text { Shanghai \& } \\
\text { Jiangsu }\end{array}$ & 3,69 & 2,05 & 2,15 & Guizhou & 3,40 & 1,67 & 1,60 \\
\hline Jiangsu & 2,42 & 0,84 & 0,77 & Yunnan & 8,78 & 3,67 & 2,77 \\
\hline Zhejiang & 1,79 & 0,65 & 0,75 & Tibet & 3,73 & - & - \\
\hline Anhui & 1,47 & 0,63 & 0,56 & Shanxi & 4,76 & 2,23 & 2,36 \\
\hline Fujian & 1,46 & 0,82 & 0,90 & Gansu & 4,81 & 2,02 & 1,90 \\
\hline Jiangxi & 3,21 & 1,24 & 1,12 & Qinghai & 7,47 & - & - \\
\hline Shandong & 1,05 & 0,49 & 0,48 & Ningxia & 1,84 & 0,98 & - \\
\hline Henan & 2,06 & 0,87 & 0,75 & Sinkiang & 5,78 & 2,10 & 1,70 \\
\hline Hubei & 7,63 & 2,87 & 2,42 & & & & \\
\hline
\end{tabular}

Tabla 2 - Índice de primacía urbana de cada provincia, índice de cuatro ciudades e índice de once ciudades, 2000

Fecha de los datos: CSSB, 2001, 2000 Censo de población de China. Fuente: Each province's urban primacy index, four city index and eleven city index, 2000 (Zhuoyong, 2008, p. 17, Table 8).

A partir de este estudio se pueden describir los siguientes indicadores:

IPT: IPT=P1 / (Suma Población total). 
El indicador de primacía utilizado resulta de dividir la población total de la primera ciudad sobre la población nacional y se le denominó Índice de Población Total (IPT) (Cuervo González, 2004, p. 82).

S2: S2=P1/P2. Donde P1 es la población de la primera ciudad.

S4: $\mathrm{S} 4=\mathrm{P} 1 /(\mathrm{P} 2+\mathrm{P} 3+\mathrm{P} 4)$. Se obtiene del cociente entre la población de la primera ciudad más poblada y la sumatoria de la población de las tres ciudades que le siguen en el ranking.

S11: $\mathrm{S} 11=2 \mathrm{P} 1 /(\mathrm{P} 2+\mathrm{P} 3+\mathrm{P} 4+\mathrm{P} 5+\mathrm{P} 6+\mathrm{P} 7+\mathrm{P} 8+\mathrm{P} 9+\mathrm{P} 10+\mathrm{P} 11)$. Se calcula el cociente entre la población de la ciudad más poblada y la suma de las diez ciudades que le siguen en el ranking.

Interpretación de valores: Cuando $S 2=2, S 4=1, S 11=1$, la distribución por tamaño es consistente con la llamada regla rango-tamaño. Cuando $\mathrm{S} 2>2(\mathrm{~S} 2<2), \mathrm{S} 4>1(\mathrm{~S} 4<1), \mathrm{S} 11>1(\mathrm{~S} 11<1)$, el sistema urbano presenta una tendencia a la concentración (dispersión) lo que significa que hay más ciudades de gran tamaño (pequeño tamaño) de las que prevé la regla rango-tamaño (Zhuoyong, 2008, p. 17) ${ }^{6}$ citado por (Alfonso-Roa, 2012, p. 16).

\section{Obtención de datos 2005-2020}

Los datos tabulados son de acceso abierto y corresponden a la información generada por el Departamento Administrativo Nacional de Estadística (DANE); Las proyecciones corresponden a estimaciones basadas en los indicadores demográficos de mortalidad, fecundidad y migraciones y la evolución del índice de importancia económica. A partir de los datos disponibles se generó la información asociada a los siguientes aspectos:

Poblacionales: Los datos poblacionales se tomaron de las estadísticas de demografía y población (DANE, 2005). Los años de consulta fueron cada 10 años: 1985, 1995, 2005, 2015, 2020 y se tomaron como referencia solamente los datos de población de la Cabecera incluyendo ciudades y municipios.

Económicos: Los datos se recuperaron del DANE $(2015,2017)$ a partir del Indicador de Importancia Económica Municipal que contiene la distribución del valor agregado departamental, entre los municipios que lo conforman.

Años de consulta disponibles: 2011, 2012, 2013, 2014, 2015.

Institucionales: La información de aspectos institucionales, se elaboró a partir de la información de la investigación realizada sobre estatalidad por Alfonso-Roa (2018). Estos datos muestran la presencia de las instituciones en cada uno de los municipios de la RAP.

\section{Resultados}

De acuerdo con lo expresado en la metodología, se realizaron los cálculos estadísticos para identificar los índices de primacía urbana en la RAPE Región Central y en la RAP Pacífico a partir de las factores poblacionales, económicos y estatales.

\section{Factores poblacionales}

Los resultados obtenidos del análisis de los factores poblacionales para RAPE-RC se presentan en la Tabla 3.

\footnotetext{
${ }^{6}$ Where Pi is the population of the ith largest city. When $\mathrm{S} 2=2, \mathrm{~S} 4=1, \mathrm{~S} 11=1$, the size distribution is consistent with the so-called rank-size rule. When $S 2>2(S 2<2), S 4>1(S 4<1), S 11>1(S 11<1)$, urban system present with the trend of concentration (disperse) which means that there are more large-size (small-size) cities than is predicted by the rank-size rule.
} 
Tabla 3 - Indicadores de primacía poblacional de la RAPE Región Central

\begin{tabular}{ccccc}
\hline Ind_Prim & $\mathbf{1 9 8 5}$ & $\mathbf{1 9 9 5}$ & $\mathbf{2 0 0 5}$ & $\mathbf{2 0 1 5}$ \\
\hline IPT & $65,6 \%$ & $67,2 \% \uparrow$ & $65,6 \% \downarrow$ & $65,1 \% \downarrow$ \\
S2 & 13,01 & $14,41 \uparrow$ & $14,57 \uparrow$ & $15,03 \uparrow$ \\
S4 & 6,63 & $6,20 \downarrow$ & $5,59 \downarrow$ & $5,28 \downarrow$ \\
S11 & 7,88 & $7,68 \downarrow$ & $7,10 \downarrow$ & $6,81 \downarrow$ \\
\hline
\end{tabular}

Fuente: Elaboración propia con datos de DANE (2005).

De acuerdo con la interpretación propuesta por Zhuoyong (2008) los municipios que conforman la RAPE-RC, se ubican entre las más concentradas ya que los índices S2, S4 y S11 superan los umbrales indicados. Cuando $S 2>2$, S4 $>1$ y $S 11>1$, se entiende que el sistema urbano presenta una tendencia a la concentración.

Específicamente se analiza que, siendo Bogotá la primera ciudad y la segunda Ibagué, permanecen constantes durante los cuatro períodos observados. El índice S2 presenta una tendencia creciente, lo que significa que las ciudades principales de la RAPE-RC se concentran más. En este caso, los umbrales superan notablemente estos indicadores y para el caso de S2 ratifica el crecimiento de la primacía poblacional de Bogotá (Figura 2).

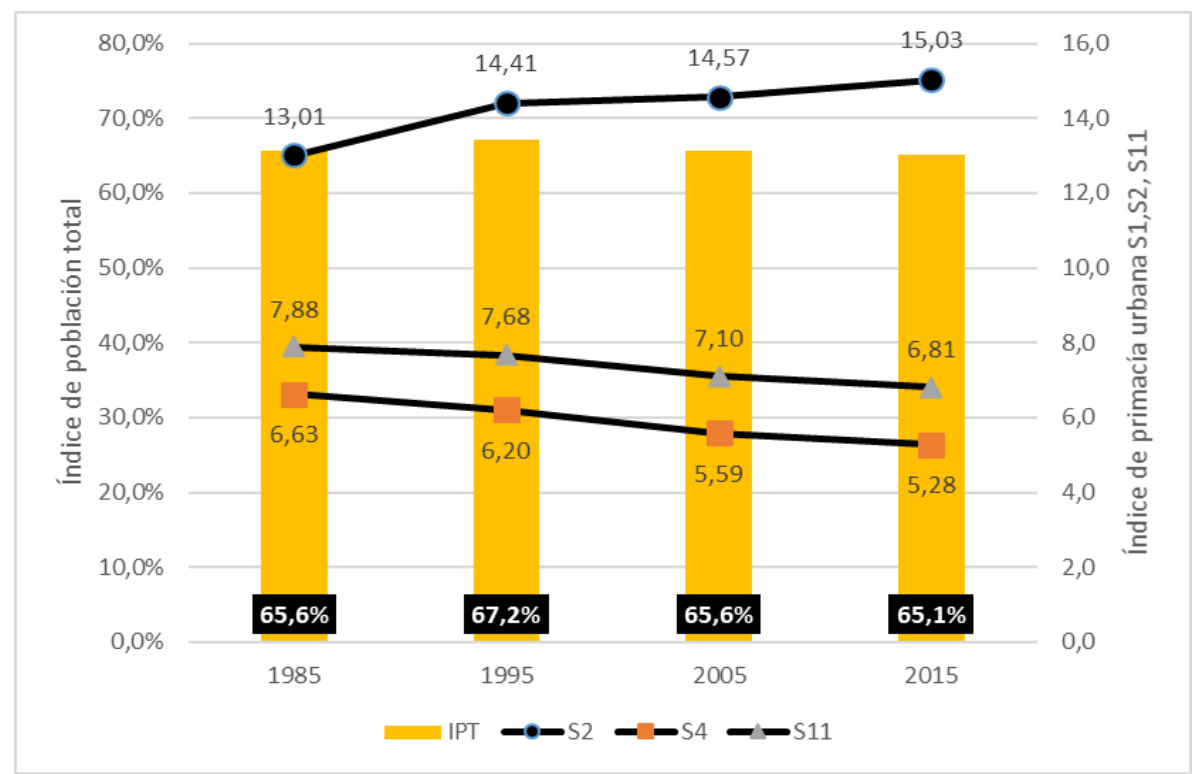

Figura 2 - Primacía poblacional RAPE Región Central. Fuente: Elaboración propia con datos DANE.

Factores poblacionales en la RAP Región Pacífico (RAP-RP) se presentan en la Tabla 4.

Tabla 4 - Indicadores de primacía poblacional de la RAP Región Pacífico

\begin{tabular}{ccccc}
\hline Ind_Prim & $\mathbf{1 9 8 5}$ & $\mathbf{1 9 9 5}$ & $\mathbf{2 0 0 5}$ & $\mathbf{2 0 1 5}$ \\
\hline IPT & $40,5 \%$ & $42,7 \% \uparrow$ & $41,6 \% \downarrow$ & $41,0 \% \downarrow$ \\
S2 & 6,27 & $6,79 \uparrow$ & $6,67 \downarrow$ & $6,38 \downarrow$ \\
S4 & 2,38 & $2,62 \uparrow$ & $2,50 \downarrow$ & $2,38 \downarrow$ \\
S11 & 2,32 & $2,53 \uparrow$ & $2,44 \downarrow$ & $2,38 \downarrow$ \\
\hline
\end{tabular}

Fuente: Elaboración propia con datos de DANE (2005).

La ciudad principal es Cali (Valle del Cauca) a lo largo de todo el periodo; la segunda es Pasto (Nariño) la cual fue desplazada por Buenaventura en el 2015. En el caso de la cuarta ciudad fue Buenaventura (Valle del Cauca) en 1985 y su ascenso fue gradual hasta ubicarse en el segundo puesto. La cuarta posición fue ocupada por Palmira (Valle del Cauca) desde 1995. Y para el caso de la posición 11 es mucho más dinámica inicia en 1985 con Yumbo (Valle del Cauca), entre 1995 y 2005 es Ipiales (Nariño) y cierra el periodo con Guadalajara de Buga (Valle del Cauca) (Figura 3). 


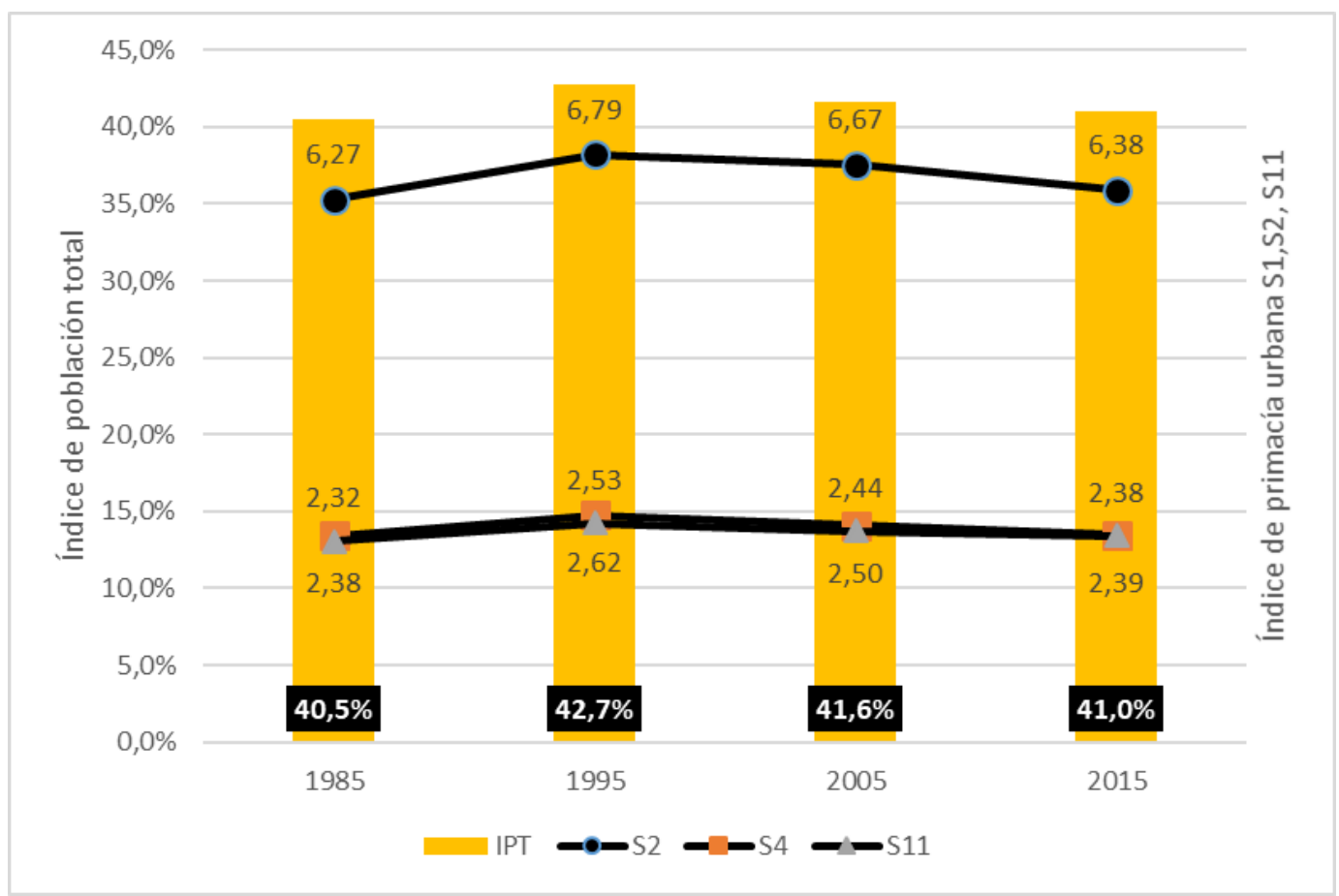

Figura 3 - Primacía poblacional RAP Pacífico. Fuente: Elaboración propia con datos DANE.

El análisis de conformación de cada uno de los departamentos de la RAP-RP varía, teniendo en cuenta que

[...] la ciudad más grande sólo debe ser 2 veces más grande que la segunda ciudad más grande, la segunda ciudad más grande sólo debe ser 3 veces como la tercera ciudad más grande, y así sucesivamente (Cuervo González, 2004, p. 78).

Las Figuras 4 a 7 se organizan de mayor a menor en relación con la concentración poblacional.

Para el caso del Valle del Cauca (Figura 4) se evidencia una fuerte concentración en relación a la segunda ciudad, pero es de anotar que gradualmente y según las cifras del DANE este fenómeno está en reducción. Mientras tanto S4 y S11 todavía evidencian un alto grado de concentración.

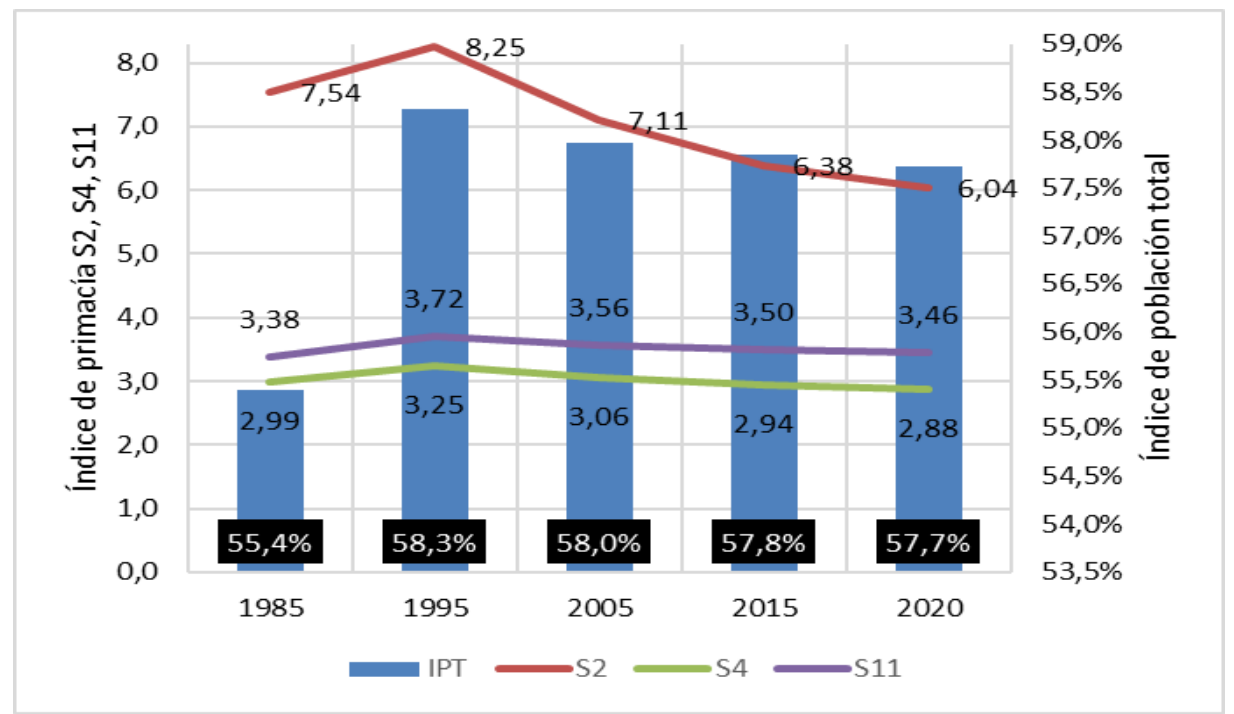

Figura 4 - Índices de primacía poblacional Valle del Cauca. Fuente: Elaboración propia con datos DANE.

En el caso del Chocó (Figura 5) se evidencia una tendencia a la diminución de la concentración. 


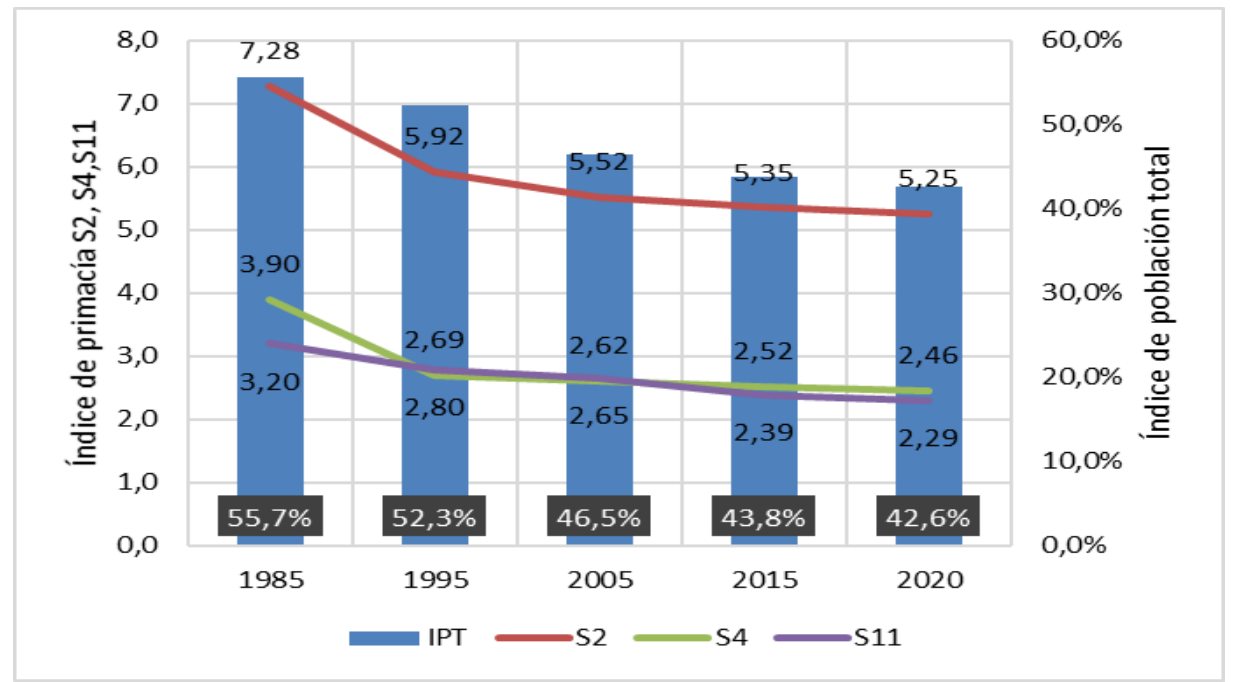

Figura 5 - Índices de primacía poblacional Choco. Fuente: Elaboración propia con datos DANE.

El Departamento del Cauca (Figura 6) es el que presenta una mayor variación entre los lapsos de tiempo seleccionados.

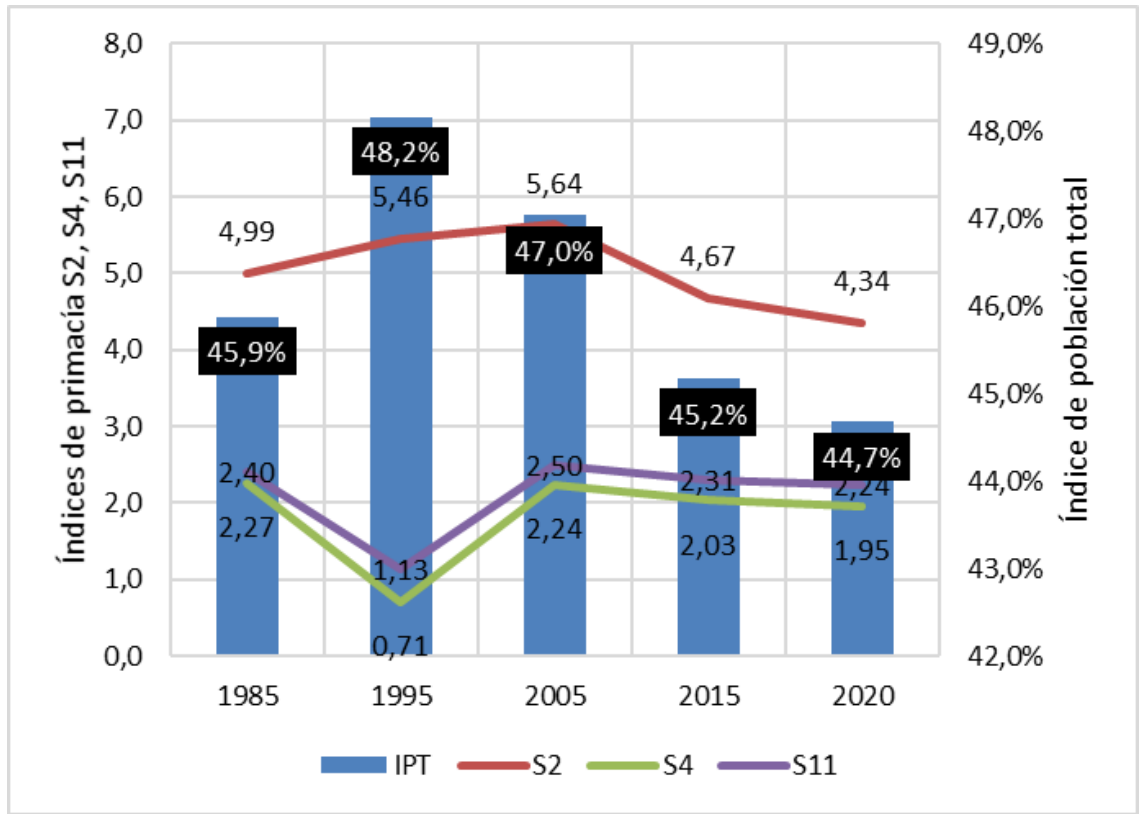

Figura 6 - Índices de primacía poblacional Cauca. Fuente: Elaboración propia con datos DANE.

En el caso del departamento de Nariño (Figura 7) es el que presenta una mayor homogeneidad entre la distribución de la población, y además se evidencia una tendencia hacia la contraurbanización (Berry, 1976; Ferrás, 2007), sin embargo, todavía los valores son muy altos a los deseados. 


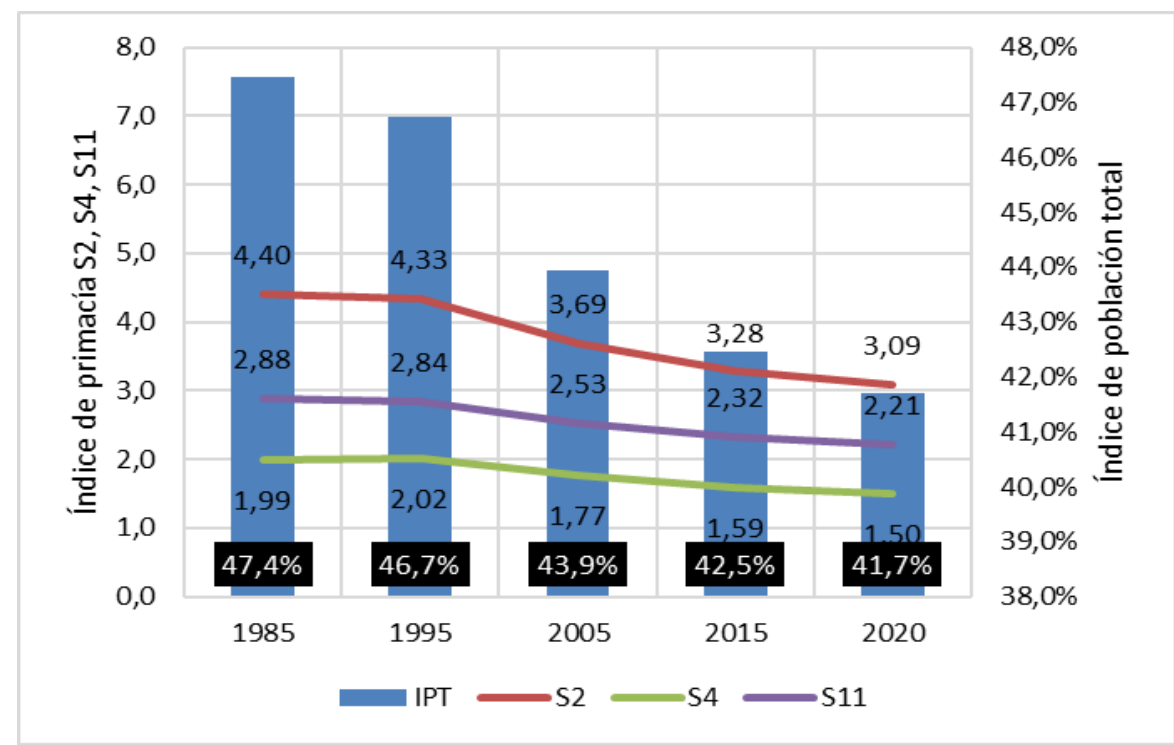

Figura 7 - Índices de primacía poblacional Nariño. Fuente: Elaboración propia con datos de DANE (2005).

En general se puede destacar la homogeneidad que presenta el Índice de Población Total (IPT) (Cuervo González, 2004, p. 82) a lo largo del tiempo y en la mayoría de los departamentos se mantiene en el rango de 0,45 hasta 0,58, siendo el Valle quien presenta los valores más altos.

Los resultados del índice de primacía poblacional en las RAP se comparan en la Figura 8. Esta dinámica de crecimiento permite observar la primacía de las poblaciones del Valle del Cauca, frente a los demás departamentos. El segundo lugar lo ocupa Nariño, pero con una escasa participación, su capital Pasto fue desplazada del segundo puesto nuevamente por el Valle del Cauca (Buenaventura). El departamento del Cauca ubica su capital (Popayán) en el 5 puesto (1985 a 2005) pero de manera ascendente hacia el 4 puesto. Dentro de la conformación de la RAP-P también está el departamento de Choco, el cual ubica a su capital Quibdó en la posición 9 en la cual se mantiene estable, pero se prevé que para el 2020 pase a la posición 11. La segunda población del Chocó es Istmina la cual se ubica en la posición 27, generando una escasa participación del Chocó en la RAP-P.
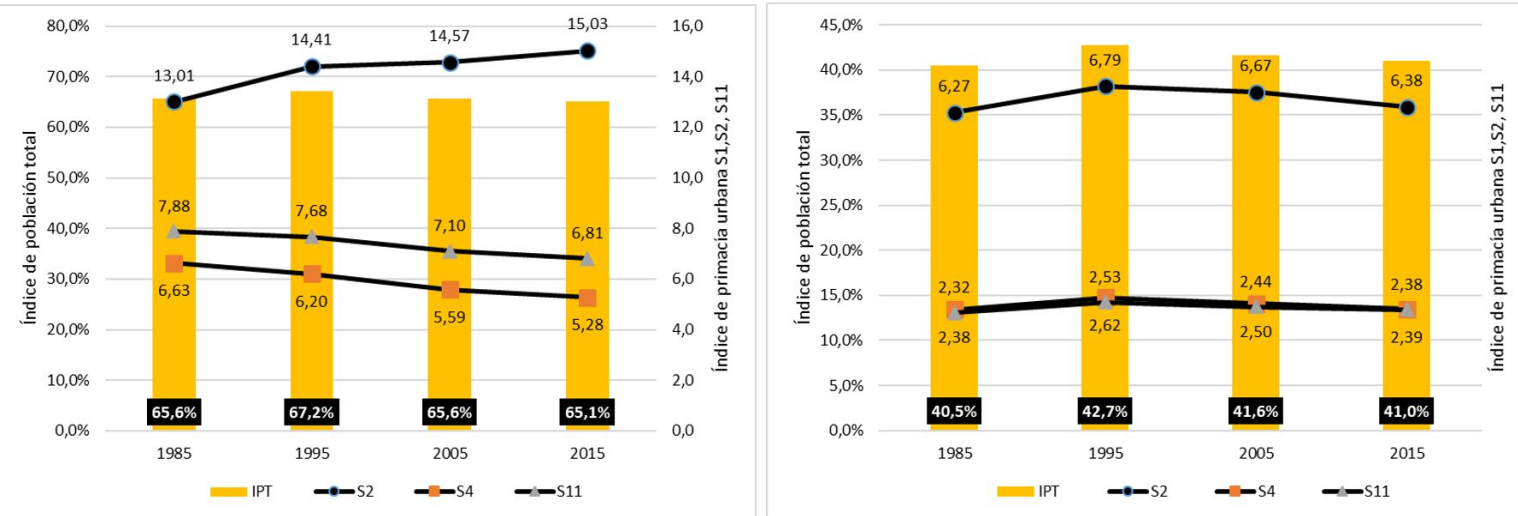

Figura 8 - Comparación de los índices de primacía poblacional en la RAPE-RC y RAP-P. Fuente: Elaboración propia (2017), con datos de DANE (2005).

Como se observa en la Figura 8, la RAP-P tiene índices de primacía menores a los de la RAPE-RC7, ubicándose casi en la mitad para el caso de S2, S4 y S11. La tendencia de crecimiento poblacional es casi estable para la RAP-P, mientras que la dinámica de la RAPE-RC es muy diversa, mientras la capital Bogotá

\footnotetext{
${ }^{7}$ Contar con Bogotá dentro de la RAPE-RC, hace que los desequilibrios sean más evidentes.
} 
incrementa su primacía frente a la S2 Ibagué (Tolima), las S4 Y S11 se mantienen estables, pero disminuyendo la concentración poblacional.

Lo anterior, pone en evidencia el desequilibrio territorial existente entre cada uno de los departamentos y entre las ciudades, que a pesar de lograr ubicarse entre las 11 ciudades principales se evidencia una concentración poblacional muy diferenciada. (El listado completo con fecha 2015, se puede consultar en el Anexo A).

\section{Factores económicos}

Tabla 5 - Índice de primacía económica RAPE Región Central

\begin{tabular}{cccccc}
\hline Ind_Prim & $\mathbf{2 0 1 1}$ & $\mathbf{2 0 1 2}$ & $\mathbf{2 0 1 3}$ & $\mathbf{2 0 1 4}$ & $\mathbf{2 0 1 5}$ \\
\hline IPT & $60 \%$ & $60 \% \triangleright$ & $60 \% \triangleright$ & $62 \% \uparrow$ & $64 \% \uparrow$ \\
S2 & 15,91 & $9,64 \downarrow$ & $9,61 \downarrow$ & $11,96 \uparrow$ & $19,58 \uparrow$ \\
S4 & 7,26 & $5,46 \downarrow$ & $5,40 \downarrow$ & $6,17 \uparrow$ & $8,23 \uparrow$ \\
S11 & 7,40 & $6,17 \downarrow$ & $6,14 \downarrow$ & $6,86 \uparrow$ & $8,40 \uparrow$ \\
\hline
\end{tabular}

Fuente: Elaboración propia con base en Indicador de importancia económica municipal DANE.

El Índice de primacía económica para la RAPE-RC (Tabla 5) indican que el S2 presentó un comportamiento dinámico durante el período analizado, dado que en los primeros años se redujo y a partir del 2014 se incrementó notablemente, lo cual es indicativo de una tendencia a la concentración económica en la primera ciudad, mostrando una tendencia al incremento en la primacía económica de Bogotá. Esta misma tendencia se repite para los índices S4 y S11, donde S4 y S11 son mayores a 1 e indican igualmente una tendencia a la concentración.

Tabla 6 - Índice de primacía económica RAP Pacífico

\begin{tabular}{cccccc}
\hline Ind_Prim & $\mathbf{2 0 1 1}$ & $\mathbf{2 0 1 2}$ & $\mathbf{2 0 1 3}$ & $\mathbf{2 0 1 4}$ & $\mathbf{2 0 1 5}$ \\
\hline IPT & $34 \%$ & $34 \% \diamond$ & $34 \% \diamond$ & $35 \% \uparrow$ & $34 \% \downarrow$ \\
S2 & 5,17 & $5,61 \uparrow$ & $4,89 \downarrow$ & $4,94 \uparrow$ & $4,70 \downarrow$ \\
S4 & 2,04 & $2,14 \uparrow$ & $1,99 \downarrow$ & $2,02 \uparrow$ & $1,97 \downarrow$ \\
S11 & 2,08 & $\underline{\mathbf{0 , 9 6} \downarrow}$ & $2,01 \uparrow$ & $\underline{\mathbf{0 , 9 4} \downarrow}$ & $2,03 \uparrow$ \\
\hline
\end{tabular}

Fuente: Elaboración propia con base en Indicador de importancia económica municipal DANE.

Las cifras de los índices de primacía económica de la RAP Pacífico (Tabla 6) indican que los índices S2 y S4, aunque menores que los obtenidos para la RAPE-RC, muestran una primacía económica de Cali como primera ciudad de esta región, pero cuando se mira en relación a las 10 primeras ciudades, se verifica un proceso de desconcentración, es decir, pérdida de la primacía, lo que hace un sistema urbano en términos económicos más equilibrado y con una oferta industrial en aumento.

Por lo tanto, entre la ciudad 5 y 11, se observa un crecimiento acelerado (Figura 9) y entre 2012 y 2013, las ciudades con más crecimiento económico fueron Yumbo y Popayán, en tanto que entre 2014 y 2015, las que mejor desempeño económico fueron Buga y Candelaria. 


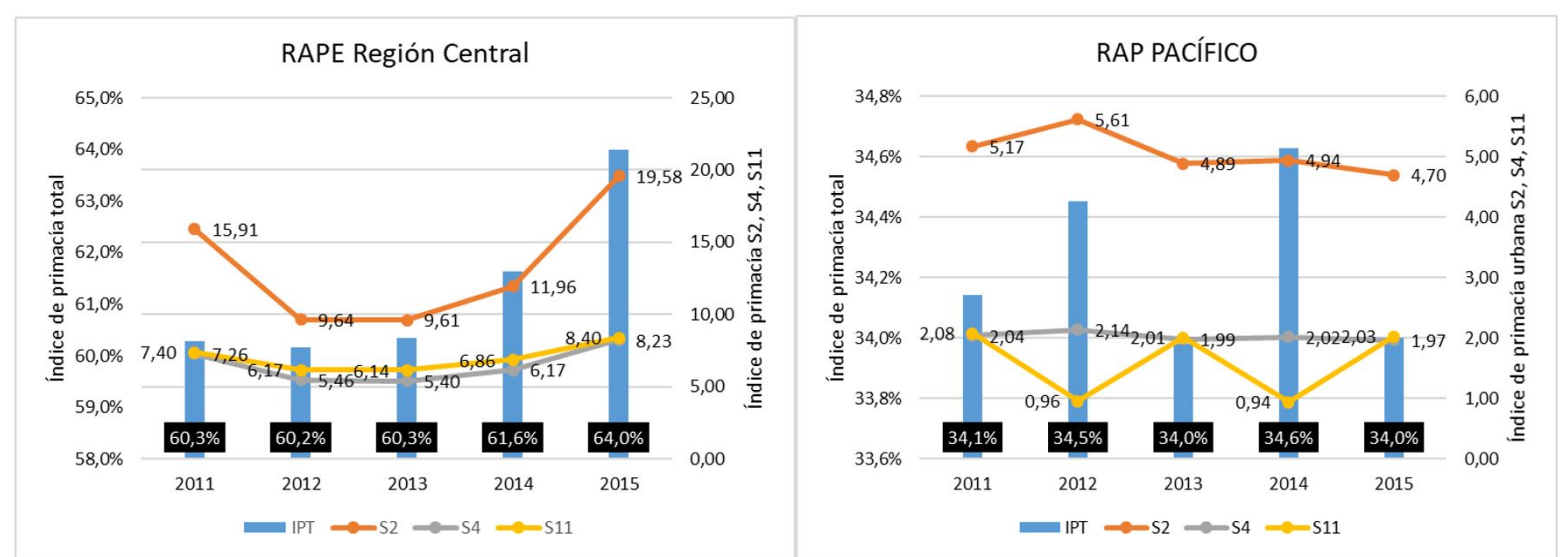

Figura 9 - Comparativo primacía económica RAPE Región Central y RAP Pacífico. Fuente: Elaboración propia con base en Indicador de importancia económica municipal DANE.

Al observar las dos gráficas de la Figura 9, se evidencia que en ambos casos hay una tendencia estable en el comportamiento del IPT, es decir, en la RAPE-RC es constante la primacía de Bogotá sobre el resto de los municipios en un 60\%, al igual que en la RAP Pacífico, con alrededor del $30 \%$, lo cual, en ambos casos concentra la primacía económica en la primera ciudad en cada región, es decir, Bogotá y Cali. Sin embargo, los resultados en la RAPE-RC muestran unos valores notablemente mayores que los de la RAP-P, especialmente, en el índice S2 que compara el indicador de importancia económica municipal en ambas regiones. Así mismo, vale decir que:

[...] "Se entiende por importancia económica el peso relativo que representa el Producto Interno Bruto de cada uno de los municipios dentro de su departamento" y, teniendo en cuenta que a nivel municipal no es posible la medición de un PIB. La expresión "peso relativo" se define como la distribución del valor agregado del departamento entre cada uno de sus distritos y municipios ubicados en su jurisdicción, a partir de una estructura obtenida con indicadores sectoriales directos e indirecto (DANE, 2015, p. 6) (comillas del texto original).

Así, el peso que tiene Bogotá en términos económicos resulta incomparable con otras entidades territoriales, siendo el doble del generado en Cali. Adicionalmente, al interior de cada RAP la primera ciudad marca la primacía económica pero que a modo de ejemplo, el Valle del Cauca tiene otros municipios como Yumbo que demuestran gran relevancia en el desarrollo económico.

\section{Factores estatales}

El índice de estatalidad es aquel que mide la presencia de las entidades del Estado en el territorio, tal como lo explica Alfonso-Roa (2018, p. 220):

La localización de las entidades estatales en el territorio le significa a ese poder tener presencia para difundir sus políticas, ejecutar los programas y asignar los recursos, así como para conformar el aparato de Estado. Pero esa presencia no significa necesariamente que el Estado ejerza efectivamente el control territorial [...] En términos del poder para la toma de decisiones asimilado por la presencia del Estado, Bogotá es 17,4 veces superior a Medellín, 8,4 veces superior a Medellín, Cali y Villavicencio, y 7,9 veces superior al acumulado en las diez siguientes ciudades de la jerarquía estatal colombiana, entre las que Barranquilla ocupa el quinto lugar.

Tomando como base en esta definición, se calcularon los índices de primacía relacionados con la estatalidad (Tabla 7): 
Tabla 7 - Índices de estatalidad en la RAPE Región Central y la RAP Pacífico

\begin{tabular}{cccc}
\hline \multicolumn{2}{c}{ Primacía RAPE Región Central } & \multicolumn{2}{c}{ Primacía RAP Pacífico } \\
\hline Ind_Prim & 2014 & Ind_Prim & 2014 \\
IPT & $75,6 \%$ & IPT & $4,89 \%$ \\
S2 & 32,84 & $\mathbf{S 2}$ & 1,55 \\
S4 & 15,66 & $\mathbf{S 4}$ & $\mathbf{0 , 7 8}$ \\
S11 & 19,84 & $\mathbf{S 1 1}$ & $\mathbf{1 , 0 6}$ \\
\hline
\end{tabular}

Fuente: Elaboración propia con base en los índices de estatalidad (Alfonso-Roa, 2018).

En este índice, es notable el desequilibrio que existe entre la RAPE Región Central y la RAP Pacífico, donde la primera evidencia una concentración marcada en relación con la segunda, dada la poca presencia del Estado en los municipios de la RAP Pacífico, donde los índices $\mathrm{S}_{2}$ y $\mathrm{S}_{4}$ definen una situación de dispersión o desconcentración de las entidades gubernamentales en la región. Es decir, entre las primeras cuatro ciudades hay una distribución más equitativa de la presencia del Estado, sobre los demás municipios analizados.

\section{Discusión}

A partir de los resultados obtenidos de la identificación, cálculo y análisis de los indicadores de primacía poblacional, económica y estatal, y la comparación entre la RAPE Región Central y la RAP Pacífico se evidenciaron desequilibrios regionales los cuales mostraron heterogeneidades, desbalances muy marcados y persistentes en el tiempo, adicionalmente, al interior de estas también son evidentes los rezagos entre los municipios y la primera ciudad de cada región (Bogotá y Cali). Esto se da a pesar de ser dos de las regiones más prosperas del país y que albergan las principales ciudades capitales. Se considera que una correcta implementación y puesta en marcha de las RAP, logrará la integración de los municipios menos favorecidos para trabajar de forma integral con otros municipios y a su vez permitirá desarrollar proyectos de alcance regional y no solamente local o departamental y de esa manera reducir los desequilibrios territoriales. No obstante, dado su reciente creación, es muy pronto para evaluar los impactos que puedan tener.

Bajo este panorama es importante identificar los ejes estratégicos planteados en cada RAP (Tabla 8), con el fin de identificar si las orientaciones pueden ayudar a la construcción de unas regiones más equilibradas.

Tabla 8 - Ejes estratégicos de desarrollo de las RAP

\begin{tabular}{ll}
\hline \multicolumn{1}{c}{ RAPE Región Central } & \multicolumn{1}{c}{ RAP Pacífico } \\
\hline 1. Sustentabilidad ecosistémica y manejo de riesgos. & 1. Riqueza colectiva: agua, biodiversidad y cultura. \\
2. Infraestructuras de transporte, logística y servicios & 2. Cierre de brechas para la equidad social. \\
públicos. & 3. Infraestructura. \\
3. Competitividad y proyección internacional & 4. Conectividad para la competitividad. \\
4. Soberanía y seguridad alimentaria & 5. Seguridad alimentaria. \\
5. Gobernanza y Buen Gobierno. & 6. Desarrollo económico. \\
& 7. Paz y posconflicto. \\
& 8. Gobernanza y desarrollo institucional. \\
\hline
\end{tabular}

Elaboración propia a partir de la información disponible en cada página web (Región Central, 2004; RAP-Pacifico, 2018).

Llama la atención que cada una las RAP determinaron ejes estratégicos teniendo en cuenta pilares que responden al desarrollo y la competitividad, pero no se hacen explicitas las características propias de cada región, entendiendo que el país goza de una diversidad vista desde el punto de vista regional.

Se considera que esta situación no es desconocida por el gobierno nacional, a modo de ejemplo está la Propuesta metodológica para el cierre de brechas territoriales plateada en el año 2014 por el Departamento Nacional de Planeación que propone diez variables que abarcan los sectores de: 
educación, salud, acueducto, vivienda e institucional entre otros (DNP, 2014b, p. 3). Con esta metodología se identifican unas tipologías que reconocen la heterogeneidad tanto en el ámbito municipal como departamental. Con los resultados se reiteran las divergencias y desbalances entre regiones, municipios y departamentos y esto sirve como punto de partida para identificar la necesidad de la intervención del Estado en aras de reducir los desequilibrios regionales. En este sentido, se deberían generar diversos incentivos para promover nuevas dinámicas de ocupación.

Del reconocimiento de los instrumentos de ordenamiento territorial, se colige que no hay evidencias efectivas sobre la reducción de los desequilibrios. Entonces, es necesario hacer seguimiento a la implementación de las RAP para determinar si es con base en el "trabajo colaborativo" que se espera que los más rezagados logren equipararse a los mejor ubicados.

Según la Ley 388/97, el artículo 7º señala que la Nación debe dar lineamientos para

[...] garantizar la equitativa distribución de los servicios públicos e infraestructura social de forma equilibrada en las regiones y la conservación y protección de áreas de importancia histórica y cultural, así como los demás temas de alcance nacional, de acuerdo con sus competencias constitucionales y legales (Colombia, 1997, Art. 7).

Sin embargo, la ley se queda corta porque no especifica cómo se alcanzará este objetivo. Adicionalmente, el Artículo 110 menciona que es competencia del Gobierno Nacional formular la Política Nacional Urbana, que garantice "el desarrollo equilibrado y eficiente de los centros urbanos", y que debe abarcar los lineamientos y parámetros para establecer los planes y fuentes de financiación. No obstante, la Política Nacional Urbana sólo habla del equilibrio en las zonas urbanas, pero no define específicamente cómo se logrará la articulación a nivel regional y nacional.

Con esto se muestra que a pesar que la Ley 388/97, tiene como uno de los pilares la búsqueda por la igualdad y equilibrio territorial, no son suficientes los planteamientos que presenta en los dos artículos expuestos, sino que se debe tener un enfoque diferencial de acuerdo con las potencialidades de cada región. En este sentido, se asume que una de las funciones de las RAP se relaciona con la posibilidad de servir como

[...] marcos de relaciones geográficas, económicas, culturales, y funcionales, a partir de ecosistemas bióticos y biofísicos, de identidades culturales locales, de equipamientos e infraestructuras económicas y productivas y de relaciones entre las formas de vida rural y urbana, en el que se desarrolla la sociedad colombiana y hacia donde debe tender el modelo de Estado republicano unitario (Prieto y Luengas, 2011, p. 8).

\section{Conclusiones}

Se debe propender por una política orientada a lograr una mejor ocupación del espacio, pero brindando oportunidades de democracia regional y justicia espacial (Soja, 2008, p. 559-563), donde el ordenamiento territorial continúe atendiendo las particularidades del territorio en un marco de democracia participativa reconociendo los derechos y responsabilidades de los ciudadanos como partícipes de la ocupación del territorio, emprendedores de la dinámicas económicas y donde el estado debe garantizar la presencia efectiva y el acompañamiento necesario para cumplir las metas trazadas, atendiendo los requerimientos de una sociedad globalizada.

Los problemas del ordenamiento reflejados en las RAP, deben ser afrontados de manera multiescalar ya que cada elemento que la conforma se comporta de forma fractal. En cada uno de los municipios y ciudades se hacen evidentes los mismos fenómenos de primacía económica y estatal. Es por esto, que si se logra implementar con éxito la figura de las RAP, será posible pensar más allá de lo local, lo departamental y pensar en términos regionales fomentado la ocupación sostenible de territorio, para lo cual es necesario incluir dentro de los planes de ordenamiento territorial un elemento que promueva la integración regional de los municipios y departamentos que conforman cada RAP. 
Se debe trabajar en pro de la gobernanza regional, para lograr la articulación de los ámbitos local y departamental en aras de alcanzar la integración de los actores y de esta forma, tomar decisiones y formular política pública con visión de región. Esto también debe ser visto desde la idea de la "[...] construcción institucional concertada" (Soja, 2008, p. 560, citando a Scott, 1998), donde las decisiones aporten incrementar la productividad y la competitividad económica regional y así, ser un medio para alcanzar el bienestar social, y lograr una mejor distribución de la población en el territorio y de las actividades económicas, en un escenario de cooperación y no de competencia. A partir de la heterogeneidad que se da entre regiones y al interior de las estas, surge el reto ante el cual deben enfrentarse estas formas de asociación regional, para lograr el objetivo consignado en el Artículo 3ํㅡ de la Ley 1454 de 2011:

Solidaridad y equidad territorial. Con el fin de contribuir al desarrollo armónico del territorio colombiano, la Nación, las entidades territoriales y las figuras de integración territorial de mayor capacidad política, económica y fiscal, apoyarán aquellas entidades de menor desarrollo relativo, en procura de garantizar el acceso equitativo a las oportunidades y beneficios del desarrollo, para elevar la calidad de vida de la población (Colombia, 2011, Art. 3).

A partir de esto, es necesario que los municipios rezagados logren equidad en su desarrollo económico y social, aprovechando las ventajas de la asociatividad, pero sin olvidar que el ordenamiento territorial también busca el incremento de la descentralización hacia las regiones y fomenta la autonomía. Sin embargo, esta autonomía está excluida de la discusión sobre el centralismo que impera en los debates ante la falta de presencia del Estado en las regiones y el desconocimiento por la realidad local. En este escenario no es posible generalizar una política para el territorio nacional, Por el contrario, es necesario reconocer las diferencias y potencialidades de cada municipio, cada departamento, situación

[...] que ha generado un efecto perverso, agudizando la inarmonía de las regiones a partir de dos fenómenos excluyentes entre sí: la concentración y la marginalidad territoriales, que se retroalimentan históricamente, bajo la forma de divergencia regional (Barbosa \& Alegría, 2010, p. 70).

Con respecto a la Estatalidad, las asimetrías son evidentes y mostró las distancias más grandes entre la RAPE-RC y la RAP Pacífico. dada la baja presencia de las entidades del Estado en algunos municipios, especialmente del Pacífico y por el contrario se reafirma la concentración de la Región Central, tal como afirmó Alfonso-Roa (2018) “Bogotá es 17,4 veces superior a Medellín, 8,4 veces superior a Medellín, Cali y Villavicencio".

Para concluir y responder a la pregunta central: ¿Cómo el ordenamiento territorial puede contribuir a atenuar los desequilibrios territoriales? Se puede afirmar que en la formulación de los planes de ordenamiento territorial se deben incluir los factores poblacionales, para lo cual es necesario contar con información estadística pertinente, actualizada y de forma oportuna. También se debe superar la planeación local, para pasar a un ámbito supramunicipal donde la gestión urbana permita pensar a nivel regional.

Por consiguiente y a partir de los resultados se generan nuevos interrogantes relacionados con la manera de comprender las formas de ordenar el territorio: $¿$ Se puede considerar esta nuevas formas urbanas de las RAP, y específicamente la RAPE-RC como una metápolis?, en la cual la composición heterogénea, con múltiples centros urbanos y grandes desequilibrios, son aspectos que coinciden con lo expuesto por Ascher (2010, p. 63) cuando afirma que las metápolis son “[...] vastas conurbações, extensas e discontinuas, heterogéneas e multipolarizadas", en este sentido, se podría entender la RAPE-RC como una megalópolis desconcentrada que tiende, en el futuro, a convertirse en una metápolis, porque abarca un territorio que se va extendiendo a través de los departamentos que conforman, diferente a lo que puede suceder con la RAP-Pacifico, en la cual cada núcleo (departamento) tiende a concentrase en sí mismo generando mayor fragmentación y poca interdependencia, lo cual no contribuye a la "solidaridad territorial" (Ascher, 1995, p. 174).

Así mismo, se concluye que las primacías estudiadas muestran las diferencias y heterogeneidades entre regiones y que sin duda, deberían estar en coherencia con lo que se planteaba Programa de "Metrópolis en Equilibrio", de Francia en los años 70, el cual buscaba, atenuar el impacto de la ciudad que 
ejerce influencia sobre la región inmediata. En el caso de la RAPE-RC con Bogotá, cuyos efectos por ser la capital del país y el centro principal de actividades económicas, genera relaciones con las ciudades cercanas y con las lejanas, actuando como centro principal. Contrario a lo que sucede en la RAP Pacífico, donde hay varios centros en cabeza de las capitales de cada departamento.

Adicionalmente, dada la primacía de Bogotá que resulta en centralismo y concentración, se requiere pensar en un modelo que desconcentre y atenúe esta primacía que genera asimetrías con las ciudades sobre las cuales tiene influencia inmediata pero también con las del resto de la Región Central.

Ahora bien, la hipótesis planteada señaló que: la fragilidad de lineamientos en el ordenamiento territorial es evidencia de un polimetropolitanismo o una macrocefalia urbana que repercute en desequilibrios territoriales que inciden sobre la forma de ocupación del territorio, entendido así, se puede afirmar que la desconcentración es necesaria como parámetro para identificar un modelo de desarrollo territorial.

A partir de la investigación se concluye que persisten ausencias en las consideraciones del ordenamiento territorial, dado que la sola expedición de normas sobre formas de organización y ordenamiento del territorio nacional, no constituyen garantía para alcanzar los objetivos y lograr la equidad territorial. Los desequilibrios persistirán si continua el crecimiento vegetativo de la población, para contrarrestar esto, es necesario que de la mano de las nuevas formas de organización regionales, se tengan en cuenta y fomenten nuevas dinámicas demográficas que permita una mejor distribución poblacional en el territorio.

Para cerrar y retomando el marco conceptual, se coincide con Oscar Alfonso-Roa cuando señala que una de bases para el ordenamiento es "[...] el equilibrio como regla de ocupación del territorio y la ausencia de contratendencias" (Alfonso-Roa, 2014, p. 113), adicionalmente, es posible retomar la idea de contraurbanización expuesta por Ferrás $(2007$, p. 6), donde afirma que se deben producir movimientos hacia los "pequeños asentamientos urbanos y rurales" como parte de las dinámicas de la población, es decir, se pueden promover los fenómenos migratorios inversos a partir de incentivos, para reorientar la ocupación del territorio y repoblar las zonas menos densas, pero garantizando las condiciones necesarias para contar con una calidad de vida adecuada.

\section{Referencias}

Alfonso-Roa, O. (2012). El sistema de ciudades y el polimetropolitanismo en Colombia. Questiones Urbano Regionales, 1, 9-38.

Alfonso-Roa, O. (2014). Los desequilibrios territoriales en Colombia: estudios sobre el sistema de ciudades y el polimetropolitanismo (Colección Economía Institucional Urbana, No. 9). Bogotá: Universidad Externado de Colombia.

Alfonso-Roa, O. (2018). Investigación estado, metrópolis, región. Bogotá: Universidad Externado de Colombia.

Ascher, F. (1995). Métapolis, ou, l’Avenir des villes. Paris: Éditions Odile Jacob.

Ascher, F. (2010). Novos princípios do urbanismo seguido de novos compromissos urbanos: um léxico (No. 22). Lisboa: Livros Horizonte.

Barbosa, 0., \& Alegría, A. (2010). Relaciones intergubernamentales y organización territorial del estado en Colombia. Bogotá: Escuela Superior de Administración Pública, Subdirección Académica, Facultad de Investigaciones.

Berry, B. J. L. (1976). The counterurbanization process: urban America since 1970. In B. J. L. Berry (Ed.), Urbanization and counterurbanization (pp. 17-30). Nueva York: Arnold.

Buzai, G. (2014). Geografía y cartografía: vínculos actuales en apoyo a la toma de decisiones en el ordenamiento territorial. Revista do Departamento de Geografia, (spe), 3-22.

Colombia. Asamblea Nacional Constituyente. (1991, 6 de julio). Constitución Política de Colombia. Bogotá: Diario Oficial. 
Colombia. Congreso de la República. (1997, 18 de julio). Ley 388 de 1997. Por la cual se modifica la Ley 9 de 1989, y la Ley 2 de 1991 y se dictan otras disposiciones. Bogotá: Diario Oficial. No. 43091.

Colombia. Congreso de la República. (2011, 29 de junio). Ley 1454 de 2011. Por la cual se dictan normas orgánicas sobre ordenamiento territorial y se modifican otras disposiciones. Bogotá: Diario Oficial 48115. Recuperado el 30 de julio de 2016, de https://www.mininterior.gov.co/sites/default/files/noticias/cartilla_ley_organica_de_ordenamiento_territorial.pdf

Coraggio, J. L. (1987). Territorios en transición: crítica a la planificación regional en América Latina. Toluca: Universidad Autónoma del Estado de México.

Cuervo González, L. M. (2004). Desarrollo económico y primacía urbana en América Latina: una visión históricacomparativa. In Consejo Latinoamericano de Ciencias Sociales - CLACSO (Ed.), El rostro urbano de América Latina: o rostro urbano da América Latina (1. ed., pp. 77-114). Buenos Aires: CLACSO. Recuperado el 17 de mayo de 2017, de http://bibliotecavirtual.clacso.org.ar/clacso/gt/20100930123442/5p2art1.pdf

Departamento Administrativo Nacional de Estadística - DANE. (2005). Proyecciones de población: información estadística. Recuperado el 22 de junio de 2017, de https://www.dane.gov.co/index.php/estadisticas-portema/demografia-y-poblacion/proyecciones-de-poblacion

Departamento Administrativo Nacional de Estadística - DANE. (2015). Indicador de importancia económica municipal: información estadística. Recuperado el 22 de junio de 2017, de https://www.dane.gov.co/index.php/estadisticas-por-tema/cuentas-nacionales/cuentas-nacionalesdepartamentales/indicador-de-importancia-economica-municipal

Departamento Administrativo Nacional de Estadística - DANE. (2017). Indicador de importancia económica municipal. Recuperado el 22 de junio de 2017, de https://www.dane.gov.co/index.php/estadisticas-portema/cuentas-nacionales/cuentas-nacionales-departamentales/indicador-de-importancia-economica-municipal

Departamento Nacional de Planeación - DNP. (2010). Plan Nacional de Desarrollo 2010-2014. Bogotá: DNP. Recuperado el 15 de octubre de 2017, de https://www.dnp.gov.co/Plan-Nacional-de-Desarrollo/PND-20102014/Paginas/Plan-Nacional-De-2010-2014.aspx

Departamento Nacional de Planeación - DNP. (2014a). Misión sistema de ciudades: una política nacional para el sistema de ciudades colombiano con visión a largo plazo (1. ed.). Bogotá: DNP. Recuperado el 22 de junio de 2017, de https://colaboracion.dnp.gov.co/CDT/_layouts/15/WopiFrame.aspx?sourcedoc=/CDT/Vivienda Agua y Desarrollo Urbano/Sistema Ciudades/Libro-Misión Sistema Ciudades.pdf\&action=default

Departamento Nacional de Planeación - DNP. (2014b). Propuesta metodológica para el cierre de brechas territoriales. Bogotá: DNP, DDTS, Grupo de Estudios Territoriales. Recuperado el 16 de mayo de 2016, de https://colaboracion.dnp.gov.co/CDT/Desarrollo\%20Territorial/Documento\%20Brechas,\%20metodolog\%C3\%A Da\%20y\%20resultados\%20(21042015).pdf

Ferrás, C. (2007). El enigma de la contraurbanización: fenómeno empírico y concepto caótico. EURE, 33(98), 5-25. http://dx.doi.org/10.4067/S0250-71612007000100001

George, P. (1967). Geografía activa. Barcelona: Ariel.

González Pinto, A. L., \& Solorza Bejarano , J. H. (2017). El sistema de ciudades en la Región Administrativa y de Planeación Especial, Región Central (RAPE-RC), Colombia. Revista Ciudades, Estados y Política, 3(3), 23-34. Recuperado el 6 de julio de 2017, de http://revistas.unal.edu.co/index.php/revcep/article/view/57329/59725

Haesbaert, R. (2014). Viver no limite: território e multi/transterritorialidade em tempos de in-seguranca e contenção. Rio de Janeiro: Bertrand Brasil.

Harvey, D. (2014). Diecisiete contradicciones y el fin del capitalismo (1. ed). Quito: IAEN.

Lobato Correa, R. L. (1998). Espacio, un concepto clave de la geografía. In G. Uribe Ortega (Ed.), Cuadernos de geografía brasileña (Serie Cómo Pensar la Geografía, No. 1, pp. 21-46). México: Centro de Investigación Científica "Ing. Jorge l. Tamayo". 
Llano, F. (2018). La gestión urbana: enseñanza a partir de sus proyecciones como campo de conocimiento y diálogo interdisciplinar. Revista de Arquitectura (Bogotá), 20(1), 24-37. http://dx.doi.org/10.14718/RevArq.2018.20.1.861

Prieto, J., \& Luengas, E. C. (2011). La ley orgánica de ordenamiento territorial como instrumento para la integración del ordenamiento territorial y ambiental. Bogotá: Universidad Militar Nueva Granada.

Rap-Pacifico. (2018). Región Administrativa y de Planificación del Pacífico (RAP Pacífico). Recuperado el 15 de septiembre de 2017, de http://rap-pacifico.gov.co/

Región Central. (2004). Región Central - Región Administrativa y de Planeación Especial (RAPE). Recuperado el 20 de junio de 2017, de http://regioncentralrape.gov.co/

Revéiz, E. (2013). El Estado estratega para el ordenamiento territorial. Bogotá: Academia Colombiana de Ciencias Económicas.

Scott, A. J. (1998). Regions and the world economy: the coming shape of global production, competition, and political order. Oxford: Oxford University Press.

Shadowxfox. (2014, 20 October). Regiones naturales de Colombia [Mapa] CC. BY-SA/4.0. Recuperado el 24 de enero de 2019, de https://commons.wikimedia.org/wiki/File:Mapa_de_Colombia_(regiones_naturales).svg

Soja, E. W. (2008). Postmetrópolis: estudios críticos sobre las ciudades y las regiones (V. Hendel, \& M. Cifuentes, Trans.). Madrid: Traficantes de Sueños. Recuperado el 10 de noviembre de 2018, de https://www.traficantes.net/sites/default/files/pdfs/Postmetrópolis-TdS.pdf

Zhuoyong, C. (2008). Urbanization and spatial structure evolution of urban system in China. IDE Institute of Developing Economies-JETRO. Recuperado el 15 de noviembre de 2017, de http://www.idejetro.jp/English/Publish/Download/Vrf/pdf/439.pdf

Editor: Paulo Nascimento Neto

Recibido: Jun. 13, 2018

Aprobado: Feb. 19, 2019 
Anexo A - listado de municipios según primacía poblacional en el 2015

\begin{tabular}{ccccc}
\hline & \multicolumn{2}{c}{ RAPE REGIÓN CENTRAL } & \multicolumn{2}{c}{ RAP PACífICO } \\
\cline { 2 - 5 } & Departamento & Municipio & Departamento & Municipio \\
\hline S1 & Cundinamarca & Bogotá, D.C. & Valle del Cauca & Cali \\
S2 & Tolima & Ibagué & Valle del Cauca & Buenaventura \\
S3 & Cundinamarca & Soacha & Nariño & Pasto \\
S4 & Meta & Villavicencio & Cauca & Popayán \\
S5 & Boyacá & Tunja & Valle del Cauca & Palmira \\
S6 & Cundinamarca & Facatativá & Valle del Cauca & Tuluá \\
S7 & Cundinamarca & Fusagasugá & Valle del Cauca & Cartago \\
S8 & Cundinamarca & Zipaquirá & Nariño & San Andres de Tumaco \\
S9 & Boyacá & Duitama & Chocó & Quibdó \\
S10 & Cundinamarca & Girardot & Valle del Cauca & Yumbo \\
S11 & Cundinamarca & Chía & Nariño & Ipiales \\
\hline
\end{tabular}

